\title{
Integración espacial en el mercado europeo de aceite de oliva
}

\author{
J.A. Roldán Casas*, R. Dios Palomares* y J. Martínez Paz ${ }^{* * *, 1}$ \\ * Departamento de Estadística, Edif. Einstein (2ªnta), Campus de Rabanales, Universidad de Cór- \\ doba, España \\ ** Departamento de Economía Aplicada, Campus de Espinardo, Universidad de Murcia, España
}

\section{Resumen}

El objetivo de este trabajo es el estudio de la integración espacial del mercado europeo del aceite de oliva, a partir de los niveles de precios de los tres principales productores y comercializadores del producto a nivel mundial (España, Italia y Grecia) entre 1961 y 2007. El análisis se lleva a cabo aplicando la técnica multivalente de cointegracion, identificando tanto relaciones a corto como a largo plazo. Los resultados del análisis de precios muestran que esos mercados de aceite de oliva están altamente integrados, con una transmisión de precios completa de precios a largo plazo, resultando Italia el mercado líder, pese a que la producción española sea con diferencia la más elevada.

Palabras clave: Aceite de oliva, cointegración, integración de mercados, series de precios.

\section{Abstract}

\section{Spatial Integration of the European Olive Oil Market}

The aim of this paper is to study the spatial integration of the European olive oil market, from the prices of the three main producers and traders in the world (Spain, Italy and Greece) between 1961 and 2007. The analysis is carried out using multivariate cointegration techniques, identifying the short-run and the long-run relationships. The empirical results show that the three olive oil markets are highly integrated, with a complete price transmission in the long run. They also suggest that Italy is the leader market being Spain the main producer.

Key words: Olive oil, cointegration, market integration, price series.

\section{Introducción y objetivos}

España, Italia y Grecia son, además de los principales países productores de aceite de oliva, los mayores exportadores del producto a nivel mundial. Así, en las campañas de producción de los años 2005/06 a 2010/11 estos tres países aglutinan en media el $72,3 \%$ de la producción mundial de aceite de oliva y el $55 \%$ del comercio mundial de este producto, sin tener en cuenta el comercio interno de la Unión Europea, que a su vez representa algo más de las tres cuartas partes del comercio total de aceite de oliva (IOC, 2012).

1. Autor para correspondencia: jmpaz@um.es 
De los tres países, destaca España, primer productor mundial de aceite de oliva, con 700.000-800.000 toneladas al año en media en la última década (según datos de la Agencia para el Aceite de Oliva (AAO), en la última campaña 2011/2012 la producción española ascendió a 1615 miles de Tm). Con un total de 282.696.000 olivos cubriendo 2.584.564 hectáreas (Avances de superficies y producciones de cultivos: Enero 2013, MAGRAMA), su superficie cultivada representa más del $25 \%$ de la superficie olivarera mundial. Este puesto de primer productor mundial hace que España sea también el primer país exportador (874,7 miles de Tm en la campaña 2011/2012 según la AAO), vendiendo al exterior aproximadamente la mitad de su producción. De esta cantidad exportada de aceite de oliva español, aproximadamente la mitad se vende a granel (envases mayores de 5 litros) que son utilizadas por los países importadores para embotellar aceites que venden como propios (Langreo, 2002). Italia es el principal destino de este tipo de venta, (entre un $40 \%$ y un $66 \%$ en las últimas campañas) que se realiza fundamentalmente en cisternas a granel, que envasa allí y reexporta a todo el mundo, siendo Canadá, EE UU y Japón sus tres grandes mercados de exportación, en los que Italia tiene cuotas de mercado por encima del $60 \%$. Este tipo de comercialización explica el hecho de que sea Italia, y no España, la que ocupa el primer lugar en el ranking de países exportadores de aceite de oliva envasado a nivel mundial (IOC, 2012), existiendo un estructura de mercado doblemente oligopsonista: la gran distribución nacional (Langreo, 2010) y las envasadoras italianas. En este sentido, el sistema español quizá adolece de fuertes políticas comercia- les de diferenciación y de calidad (Dios y Martínez-Paz, 2011), y de planes de promoción internacional del sector que puedan generar mayores ventajas competitivas (Moral y Lanzas, 2010).

No obstante, las exportaciones españolas de aceite envasado se han incrementado de forma considerable en los últimos años (y continúan al alza), por la irrupción en el mercado mundial de nuevos importadores, como Brasil, Rusia, India o China, que han llevado a cerrar la campaña 2010/2011 con una cifra récord de ventas al exterior, el $60 \%$ de la producción nacional, destacando "especialmente el avance cosechado en los nuevos mercados, en los que España ha logrado aventajar claramente a Italia como proveedor" (Alimarket, 2011)2. Todas estas cifras ponen claramente de manifiesto las importantes interrelaciones internacionales del mercado de aceite de oliva, donde son tan importantes los aspectos comerciales y de marketing (Parras-Rosa et al., 2013) como los más estudiados de producción (Parras et al., 2003).

El objetivo de este trabajo es analizar un aspecto básico para ahondar en la compresión del funcionamiento internacional del mercado del aceite de oliva como es el de las relaciones espaciales de los precios de exportación del mismo. Para ello se analizará el mercado de los tres principales países productores a nivel mundial (España, Italia y Grecia) y se comprobará si el mecanismo de transmisión de precios es perfecto, lo que implicaría que, en el largo plazo, las variaciones en un precio se trasmiten completamente a los demás. De esta forma, se podría hablar de la existencia de un precio único y, por tanto, de un mercado eficiente.

2. Valga como ejemplo el estudio realizado para el Instituto Español de Comercio Exterior, ICEX (2012) que señala el caso de las ventas de aceite virgen extra español a China, país al que España proveyó la mitad de su consumo en 2009. Así, se exportó un volumen superior a 10 millones de $\mathrm{kg}$ que proporcionaron un valor de 33,5 millones de euros. Esto supone un aumento del 28 por ciento respecto a lo facturado durante el ejercicio anterior por este tipo de aceite en este país, calificado en 2012 como el nuevo "El Dorado" (Antelo, 2012). 
El estudio realizado se estructura en diversos apartados. En el apartado 2 se detalla la metodología aplicada para analizar la integración espacial de mercados y los datos utilizados con este fin. El apartado 3 se dedica a presentar y discutir los resultados obtenidos y finalmente, en el apartado 4 se indican las principales conclusiones que se derivan del estudio.

\section{Material y métodos}

Para alcanzar el objetivo del trabajo se aplica el enfoque metodológico propuesto por Johansen (1988), que permite distinguir las relaciones a largo y corto plazo, y permite medir el grado de integración entre los precios a la exportación en diferentes mercados separados en el espacio, determinando además si existe algún mercado líder en cuanto a la fijación de los precios se refiere y, en este caso, estudiar cómo reaccionan el resto de mercados ante variaciones del precio en el líder.

Esta metodología ha venido siendo empleada ampliamente para el estudio de la integración espacial de mercados, especialmente los de productos agroalimentarios, en todas las latitudes y escalas espaciales. Valgan como ejemplo los estudios sobre el mercado del arroz (Silvapulle y Jayasuriya, 1994), los cereales (Gil et al., 1996), el maíz (Abdulai, 2000), la carne de cerdo y cordero (Sanjuan y Gil, 2001), el algodón (Baffes y Ajwad, 2001), las hortalizas de invernadero (Martínez-Carrasco, 2004), el pescado blanco en Europa (Nielsen, 2005) o el mercado internacional del azúcar (Alonso y Arcila, 2012). Entre los trabajos citados se encuentran las principales aplicaciones del estudio de integración de precios y mercados a productos agroalimentarios en España. También cabe señalar algunos trabajos que realizan una revisión general de las metodologías y sus aplicaciones como son los artículos de Barret (2001), Fackler y Goodwin
(2001), Barret y Li (2002) y Rapsomanikis et al. (2004). Sin embargo, para el producto que se considera en el presente artículo, las publicaciones existentes no son muy numerosas, pudiendo destacar las de Gil et al. (2004) que estudian la integración de los mercados español y griego, Roldan y Dios (2008) que estudian la verificación de la ley de precio único en el mercado español del aceite de oliva, Ben Kaabia, y Gil (2008) que modelan las exportaciones de aceite de oliva en Francia y los recientes trabajos de Gutiérrez y Vega (2009) y Gutiérrez (2012) que analizan en profundidad la dinámica del mercado en origen del aceite español con técnicas de cointegración.

Se presenta en primer lugar el origen de las series de datos utilizadas y a continuación se describen los métodos econométricos utilizados en su estudio.

\section{Material: datos utilizados}

Los datos utilizados en este estudio son las series anuales de precios medios del aceite de oliva exportado por los tres países estudiados (España, Italia y Grecia) durante el período 1961 a 2007, que es el periodo disponible más largo posible con un tratamiento que asegura la homogeneidad de la serie. Con el fin de obtener un valor representativo de dichos datos se han construido tres series de precios a partir de los datos proporcionados por la Organización de las Naciones Unidas para la Alimentación y la Agricultura (FAO, 2012) relativos a las exportaciones totales, tanto en unidades monetarias (\$) como en unidades físicas $(\mathrm{kg})$ en términos nominales, de forma que el cociente de ambas permite obtener una serie de precios en $\$ / \mathrm{kg}$.

La Figura 1 recoge la evolución del precio anual de exportación del aceite de oliva en España, Italia y Grecia de 1961 a 2007 (con la notación PESPAÑA, PITALIA y PGRECIA, respectivamente), calculado en la forma indicada en el pá- 


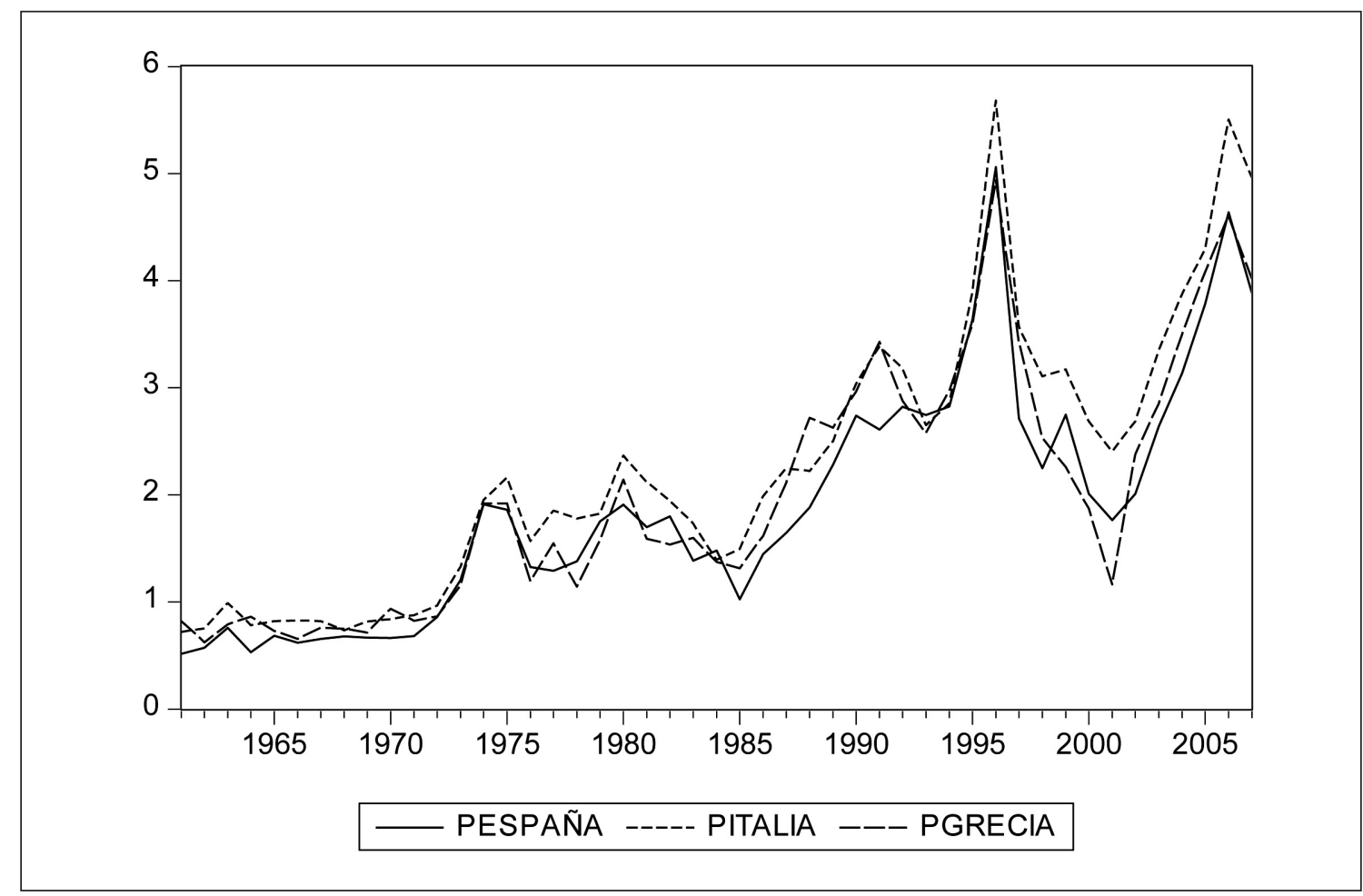

Figura 1. Evolución del precio (\$/kg) de exportación del aceite de oliva (1961-2007).

Figure 1: Evolution of olive oil export price $(\$ / \mathrm{kg})$ (1961-2007).

Fuente: Elaboración propia a partir de FAO (2012).

rrafo anterior. Como se puede observar, todas las series de precios evolucionan de manera similar presentando un crecimiento a lo largo del período considerado.

Cabe destacar una cresta atípica correspondiente a los datos del año 1995 en que la escasez de producción dio lugar a una subida espectacular en 1996 situándose el precio en torno a $5 \$ / \mathrm{kg}$ en los tres países objeto de estudio. Esta menor cosecha junto con la falta de stocks de regulación en la Unión Europea y el mantenimiento de la demanda en el consumo interno provocaron el fuerte incremento de precios. Tras esta situación puntual, se produce la recuperación de los niveles de precios anteriores permaneciendo el precio medio entre 1 y $4 \$ / \mathrm{Kg}$. No obstante, hay que señalar que en 2004 se produce un aumento importante de la cantidad exportada en los tres países y un incremento de su valor unitario. Aunque las cantidades exportadas en los años siguientes disminuyen, el precio se mantiene en los valores elevados previamente alcanzados.

Destaca el hecho de que el precio de Italia parece mantenerse casi siempre por encima de los otros dos, siendo la diferencia mayor con el precio de España. En este sentido, según se observa en la tabla 1, el precio medio de Italia a lo largo del período considerado es de $2,27 \$ / \mathrm{kg}$ mientras que el de España es 1,89 $\$ / \mathrm{kg}$. Podemos encontrar la explicación en lo 
ya comentado en la introducción de este trabajo de que gran parte del aceite exportado por Italia proviene de la gran cantidad de aceite a granel que este país importa de España para envasar y exportar como propio, lo que, por regla general, propiciaría un precio medio de exportación superior al de España.

Tabla 1. Medidas descriptivas del precio (\$/kg) del aceite de oliva (1961-2007) Table 1. Descriptive statistics for olive oil export price (\$/kg) (1961-2007)

\begin{tabular}{lccc}
\hline & PESPAÑA & PITALIA & PGRECIA \\
\hline Media & 1,897 & 2,270 & 2,009 \\
Mediana & 1,764 & 2,121 & 1,617 \\
Máximo & 5,067 & 5,681 & 4,935 \\
Mínimo & 0,517 & 0,720 & 0,623 \\
\hline Desv. Típica & 1,113 & 1,281 & 1,147 \\
\hline
\end{tabular}

Fuente: Elaboración propia.

El análisis del mecanismo de transmisión de los precios se lleva a cabo con las tres series de precios expresadas en logaritmos ${ }^{3}$ (que denotamos LPCESPAÑA, LPCITALIA y LPCGRE$\mathrm{CIA}$ ) ya que dicha transformación amortigua la amplitud de las oscilaciones de cada serie y permite alcanzar más fácilmente la estacionariedad en varianza en caso de que haya que diferenciar por no ser estacionaria.

Metodología: técnicas económetricas aplicadas

Para el análisis de las relaciones a largo plazo entre un conjunto de $p$ series, el procedimiento propuesto por Johansen (1988) parte de un modelo de Vectores Autorregresivos (VAR), cuya expresión general responde a

$$
\mathrm{Z}_{t}=\sum_{i=1}^{k} \mathrm{~A}_{i} \mathrm{Z}_{t-i}+\varepsilon_{t}
$$

donde $Z_{t}$ es el vector columna de las $p$ series que constituyen el modelo; $A_{i} i=1, \ldots, k$, son matrices $p \times p$ de coeficientes y $\varepsilon_{t}$ es el vector columna de los términos de error tal que $\varepsilon_{t}$ $\sim \operatorname{iid}(0, \Sigma)$.

Si todas las variables de $Z_{t}$ son integradas del mismo orden, siendo el orden de integración 0 o 1, el modelo (1) se puede reparametrizar en la forma

$$
\Delta \mathrm{Z}_{t}=\Pi \mathrm{Z}_{t-1}+\sum_{i=1}^{k-1} \Gamma_{i} \Delta \mathrm{Z}_{t-i}+\varepsilon_{t} \quad \text { para } k \geq 1, \quad \varepsilon_{t} \sim \mathrm{iid}(0, \Sigma)
$$

donde

$$
\begin{aligned}
& \Pi=-\left(\mathrm{I}-\mathrm{A}_{1}-\ldots-\mathrm{A}_{k}\right) \quad \text { y } \quad \Gamma_{i}^{*}=-\left(\mathrm{A}_{i+1}+\mathrm{A}_{i+2}+\ldots+\mathrm{A}_{k}\right), \\
& j=1,2, \ldots, k-1
\end{aligned}
$$

En este contexto, el teorema de representación de Granger (Engle y Granger, 1987) establece que

* si el rango de la matriz П es igual a $p$ (número total de variables en el modelo $V A R$ ), el vector $Z_{t}$ es estacionario (esto es, las variables que constituyen $Z_{t}$ son $I(0)$

* si el rango de la matriz $\Pi$ es igual a $r<p$, existe una representación de $\Pi$ tal que

$$
\Pi=\alpha \beta^{\prime}
$$

donde $\alpha$ y $\beta$ son matrices $p \times r$. La matriz $\beta$ verifica $\beta^{\prime} Z_{t} \sim /(0)$, incluso en el caso en el que $Z_{t}$ $\sim /(1)$, lo que significa que las variables que

3. Para evitar la existencia de valores negativos de la serie en logaritmos, las serie de precios fueron expresadas en centavos de $\$ / \mathrm{kg}$. 
constituyen $z_{t}$ están cointegradas, siendo los vectores de cointegración cada una de las $r$ columnas de $\beta$ (matriz de cointegración). En este caso particular, (esto es, cuando $Z_{t} \sim$ $I(1))$, el modelo (2) incorpora, además de la relación a largo plazo existente entre las variables en cuestión (a través de la matriz $\beta^{\prime} Z_{t}$ ), un mecanismo de corrección de error, de manera que las ecuaciones de dicho modelo son las ecuaciones de la relación a corto plazo entre las variables de $Z_{t}$, siendo los elementos de la matriz $\alpha$ los coeficientes de ajuste, pues miden la velocidad con la que cada variable se ajusta a la relación de equilibrio.

Según Goodwin y Schroeder (1991) la condición necesaria para que $p$ mercados separados geográficamente se consideren perfectamente integrados es que entre las series de precios existan $p-1$ vectores de cointegración, es decir, a largo plazo, los precios en los $p$ mercados se mueven en la misma dirección existiendo una única tendencia común que condiciona la evolución del resto de los precios. La condición suficiente es que cada vector de cointegración relacione una pareja de precios en la forma $(1,-1)$.

La estrategia seguida en este trabajo puede resumirse en las fases de determinación del orden de integración, especificación del modelo y estudio de las relaciones de integración, que son presentadas brevemente a continuación:

\section{Fase I: Orden de integración de las series}

Sea $P_{i t}$ la notación para la $i$-ésima serie de $Z_{t}$. Así, para determinar el orden de integración de cada serie $P_{i t}$ se aplicó la estrategia de detección de raíces unitarias que desarrollan Dios y Roldán (2006), denotada RyD, a partir de la estimación MCO del modelo

$$
\begin{aligned}
& P_{i t}=\mu+\beta t+\rho P_{i(t-1)}+\sum_{j=1}^{k} \phi_{i}\left(P_{i(t-j)}-P_{i(t-j-1)}\right)+e_{t} \\
& e_{t} \sim \mathrm{iid}\left(0, \sigma^{2}\right)
\end{aligned}
$$

donde al ser $k$ desconocido se eligió como valor de dicho parámetro aquél que minimiza el criterio de información de Schwarz.

\section{Fase II: Especificación del modelo}

Para que los contrastes de cointegración proporcionen resultados fiables es necesaria una correcta especificación del modelo (2). Así, por un lado se estableció la dinámica del modelo mediante una correcta elección del valor de $k$, para lo cual se emplearon, sobre el modelo (1), los criterios del Error de Predicción Final (EPF) de Akaike y Schwarz (Koehler y Murphree, 1988). Por otro lado, fue necesario especificar las componentes deterministas a incluir en (2), siendo lo más habitual una constante y/o una tendencia determinista lineal $t$ que podrán estar restringidas o no a las relaciones de cointegración, lo que da lugar a 5 especificaciones distintas de (2) (Johansen, 1995).

\section{Fase III: Relaciones de cointegración}

Una vez especificado correctamente el modelo (2) se determinó el número de relaciones de cointegración entre las series $Z_{t}$ de aplicando los contrastes de la traza y del autovalor máximo propuestos por Johansen (1988), los cuales se basan en la estimación de máxima verosimilitud de (2).

De la estimación máximo-verosímil de (2) se obtuvieron las estimaciones de los $r$ vectores de cointegración, lo cual permitió estimar las ecuaciones de la relación a largo plazo existente entre las series analizadas. No obstante, previo a esta especificación se contrastó la intervención de las diferentes series en la relación a largo plazo. Así, se admite la relevancia de una serie $P_{i t}$ en la relación a largo plazo si se rechaza la hipótesis:

$$
\beta_{i 1}=\beta_{i 2}=\ldots=\beta_{i r}=0
$$

es decir, si no se admite la nulidad conjunta de sus coeficientes en las $r$ relaciones de 
cointegración (columna $r$ de la matriz $\beta$ en (3)) detectadas. Esta hipótesis se contrastó con el test de la razón de verosimilitud construido a partir del modelo (2). En caso de que alguna serie no resultara relevante en la relación a largo plazo, cabía la posibilidad de excluirla del estudio y analizar la integración en un subconjunto de mercados, lo que requeriría la reestimación de (2) y, por tanto, de las relaciones a largo plazo.

Fase IV: Estudio del liderazgo en la fijación de precios

En este contexto, se realizó un estudio sobre la posible existencia de mercados que se encuentran en una situación de predominio o liderazgo frente a los demás. El que un mercado sea considerado líder implica que incide sobre el resto de mercados y no se ve afectado por los shocks específicos que se producen en ellos. Esta situación ocurre cuando para un mercado $i$ su serie de precios $P_{i t}$ es débilmente exógena en el contexto del modelo (2), o lo que es igual, cuando los coeficientes, $\alpha_{i t} j=1, \ldots, r$, (fila $i$ de la matriz $\alpha$ en (3)) son nulos.

Si las series de precios analizadas son integradas de orden 1 existiendo $r=p-1$ relaciones de cointegración entre ellas, se producirá la perfecta integración de los $p$ mercados si en cada uno de los vectores de cointegración se satisface la relación $(1,-1)$ entre las diferentes parejas de precios. Esto equivale a contrastar la hipótesis:

$$
H_{0}: \beta^{\prime}=\left[\begin{array}{cccccc}
1 & -1 & 0 & \cdots & 0 & * \\
1 & 0 & -1 & \cdots & 0 & * \\
\vdots & \vdots & \vdots & \ddots & \vdots & \vdots \\
1 & 0 & 0 & \cdots & -1 & *
\end{array}\right]
$$

En la matriz $\beta^{\prime}$ cada fila es un vector de cointegración y la última columna representa el término constante (en el caso que se incluya) en cada relación de cointegración (el símbolo * indica que el parámetro correspondiente no está restringido). En cualquier caso, las restricciones que establece (7) se contrastaron con el correspondiente estadístico de la razón de verosimilitud que se construye a partir de la estimación máximo verosímil de (2).

\section{Fase V: Relaciones dinámicas a corto plazo}

Mediante la obtención de las denominadas Funciones Impulso-Respuesta (FIR) se analizaron las interacciones dinámicas a corto plazo que caracterizan al sistema objeto de estudio. En concreto, dichas funciones muestran la respuesta de las variables (series) explicadas en el sistema ante cambios en los errores. Un cambio (shock) en una serie en un instante determinado afectará directamente a la propia serie y se transmitirá al resto de series a través de la estructura dinámica que representa el modelo VAR, proporcionando las FIR el signo, la magnitud y la persistencia de la respuesta de cada serie.

\section{Resultados y discusión}

En este apartado se presentan y discuten los resultados obtenidos de aplicar la metodología que se acaba de exponer a las series de datos descritas. En su exposición se sigue el esquema secuencial propuesto, es decir, estudio del orden de integración, análisis de integración a largo plazo y las relaciones dinámicas a corto plazo.

\section{Orden de integración de las series}

En la Figura 2, que recoge la representación del logaritmo de las series de precios en centavos de \$ por kg, se observa un deambular de las tres series alrededor de una tendencia lineal creciente, y no parece existir ningún tipo de componente cíclica. Este comporta- 


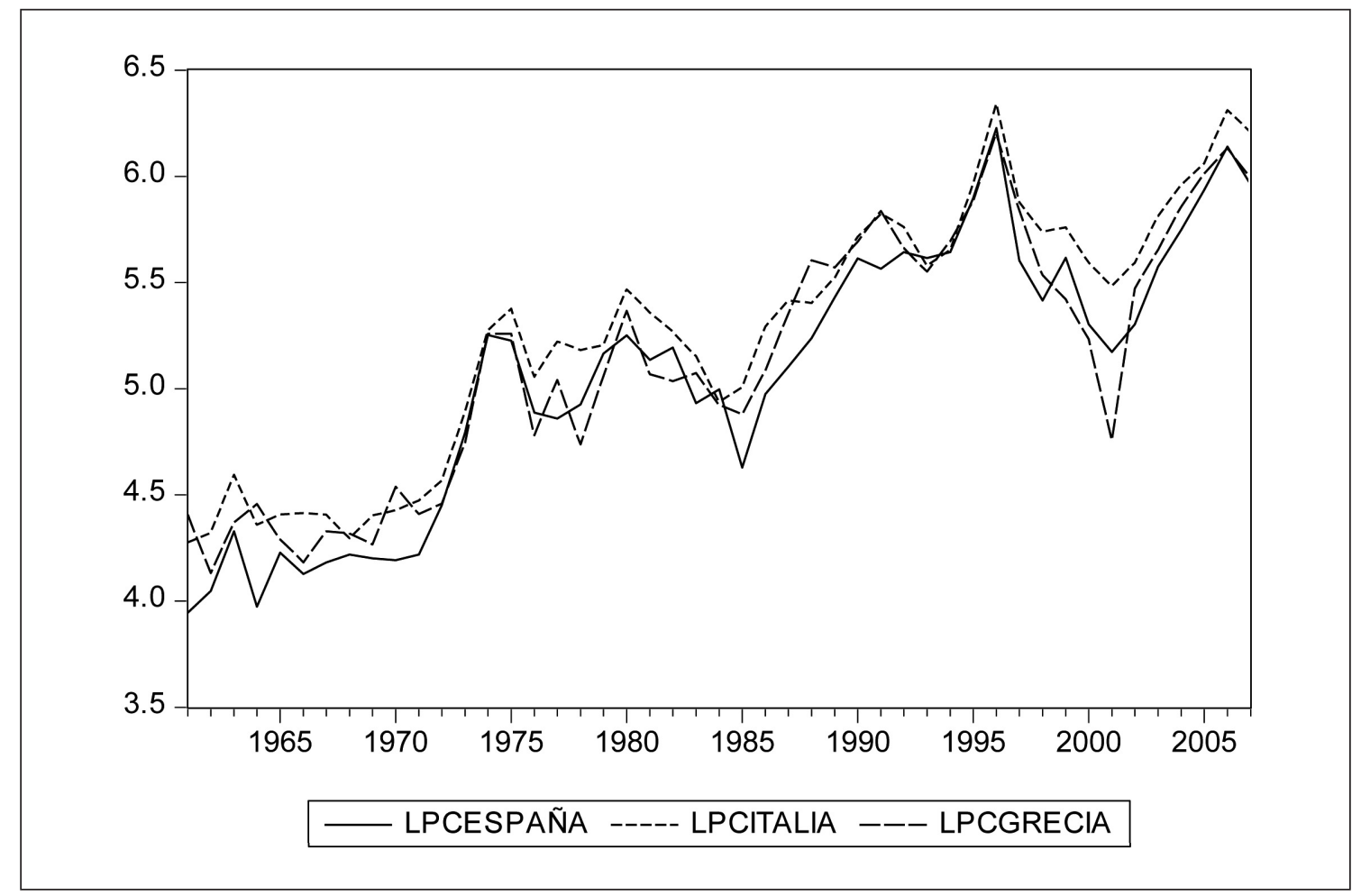

Figura 2. Evolución del logaritmo precio (cent/kg) de exportación de aceite de oliva. Figure 2. Evolution of olive oil export price (cent./kg) in logarithms (1961-2007).

Fuente: Elaboración propia a partir de FAO (2012).

miento ${ }^{4}$ es típico de series con tendencia en media y en varianza lo cual se corresponde con un mecanismo generador que presenta una raíz unitaria junto con un término constante ${ }^{5}$.

Para confirmar lo anterior se aplicó la estrategia RyD a la estimación MCO del modelo (4), cuyos resultados se recogen en la tabla 2.
Para las tres series de precios objeto de análisis el valor calculado del estadístico $t$ de $\rho$ cae en lo que la estrategia RyD denomina "zona de duda", esto es: o bien la serie tiene una raíz unitaria sin tendencia determinista lineal o es estacionaria alrededor de dicha tendencia. Esto llevó a contrastar la nulidad del coeficiente $\beta$ de la tendencia lo cual con-

4. Se realizaron los correlogramas de cada una de las series en niveles y en todos ellos se observó el comportamiento típico de una serie con raíz unitaria (caída lineal de las autocorrelaciones). Posteriormente, se realizaron los correlogramas de las series en primeras diferencias y en ellos no se encontró ningún tipo de estructura (dichos correlogramas pueden ser solicitados a los autores).

5. La presencia en el mecanismo generador de una serie de una raíz unitaria junto con un término constante induce una tendencia lineal creciente en dicha serie (Roldán, 2000). 
Tabla 2. Contrastes de raíz unitaria: estrategia RyD

Table 2. Unit root tests: RyD strategy

\begin{tabular}{|c|c|c|c|c|c|c|c|}
\hline \multirow{2}{*}{$P_{i t}$} & \multirow{2}{*}{$\begin{array}{l}\text { Elementos } \\
\text { deterministas }\end{array}$} & \multirow[b]{2}{*}{$\mathrm{k}$} & \multirow{2}{*}{$\begin{array}{l}\text { Valores calculados } \\
\text { de los estadísticos }\end{array}$} & \multicolumn{4}{|c|}{ Test de Ljung-Box* } \\
\hline & & & & $\mathrm{Q}(1)$ & $\mathrm{Q}(3)$ & $Q(12)$ & $Q(18)$ \\
\hline \multirow{3}{*}{ LPCESPAÑA } & \multirow{3}{*}{ cte y $t$} & \multirow{3}{*}{0} & $\hat{t}_{\rho}=-2,828957$ & \multirow{3}{*}{$\begin{array}{l}0,5346 \\
(0,465)\end{array}$} & \multirow{3}{*}{$\begin{array}{l}0,6674 \\
(0,881)\end{array}$} & \multirow{3}{*}{$\begin{array}{l}10,422 \\
(0,579)\end{array}$} & \multirow{3}{*}{$\begin{array}{l}19,656 \\
(0,353)\end{array}$} \\
\hline & & & $\hat{t}_{\beta}=2,38904$ & & & & \\
\hline & & & $\hat{\Phi}_{3}=4,041087$ & & & & \\
\hline \multirow{3}{*}{ LPCITALIA } & \multirow{3}{*}{ cte y $t$} & \multirow{3}{*}{1} & $\hat{t}_{\rho}=-3,353392$ & \multirow{3}{*}{$\begin{array}{l}0,0102 \\
(0,920)\end{array}$} & \multirow{3}{*}{$\begin{array}{l}0,4346 \\
(0,933)\end{array}$} & \multirow{3}{*}{$\begin{array}{l}5,0477 \\
(0,956)\end{array}$} & \multirow{3}{*}{$\begin{array}{l}10,718 \\
(0,906)\end{array}$} \\
\hline & & & $\hat{t}_{\beta}=3,062474$ & & & & \\
\hline & & & $\hat{\Phi}_{3}=3,905054$ & & & & \\
\hline \multirow{3}{*}{ LPCGRECIA } & \multirow{3}{*}{ cte y $t$} & \multirow{3}{*}{0} & $\hat{t}_{\rho}=-3,105971$ & \multirow{3}{*}{$\begin{array}{c}0,619 \\
(0,431)\end{array}$} & \multirow{3}{*}{$\begin{array}{c}0,797 \\
(0,850)\end{array}$} & \multirow{3}{*}{$\begin{array}{l}7,7445 \\
(0,805)\end{array}$} & \multirow{3}{*}{$\begin{array}{l}17,708 \\
(0,475)\end{array}$} \\
\hline & & & $\hat{t}_{\beta}=2,756464$ & & & & \\
\hline & & & $\hat{\Phi}_{3}=4,833862$ & & & & \\
\hline
\end{tabular}

Regiones de la estrategia RyD para $T=50$ al $5 \%$ :

- Para $H_{0}: \rho=1$ vs $H_{1}: \rho<1 \quad$ región de rechazo $(-\infty,-3,50) \quad$ región de aceptación $(-1,68,+\infty)$

- Para $H_{0}: \beta=0$ vs $H_{1}: \beta^{1} 0 \quad$ región de rechazo $(-\infty,-3,18)$ È $(3,80,+\infty)$ región de aceptación $(0,58,2.01)$

Las zonas de la recta real no cubiertas por ninguna de las regiones constituyen zonas de indeterminación

- Para $H_{0}: \rho=1, \beta=0 \quad$ región de rechazo $(0,0,93) \cup(6,73,+\infty) \quad$ región de aceptación $(0,93,6,73)$

* En cada celda aparece el valor calculado del estadístico $Q(i)$ de Ljung-Box para $i$ retardos y, debajo, entre paréntesis, se reproduce el $p$-valor correspondiente.

Fuente: Elaboración propia.

dujo también a una de las zonas de duda de la citada estrategia, pues el valor calculado del estadístico no es lo suficientemente elevado, en valor absoluto, para rechazar la nulidad del coeficiente $\beta$ sin que se vea afectado por la no similaridad de dicho contraste. Esto implica una duda también sobre la significación de $\beta$ en los tres casos. En este contexto la estrategia RyD propone resolver las dudas con el contraste global basado en el estadístico $\Phi_{3}$ según el cual se aceptó $\rho=1, \beta$ $=0 \mathrm{y} \alpha$ cualquiera, para las tres series de precios. En consecuencia, las tres series de precios presentan una raíz unitaria con término constante que induce en ellas una tendencia lineal como cabía observar en la Figura 1.
Análisis de las relaciones a largo plazo

a) Especificación del modelo de corrección de error

Puesto que las series han resultado integradas de orden 1, el siguiente paso es determinar si dichas series están cointegradas, es decir, contrastar si existe alguna relación de equilibrio estacionaria entre ellas sobre la base del modelo de corrección de error (2). Dicho contraste requiere una correcta especificación del modelo (2) en lo que se refiere al número $k$ de retardos óptimo y las componentes deterministas a incluir.

La tabla 3 indica que los tres criterios de información considerados establecen que el 
Tabla 3. Selección del $\mathrm{n}^{\circ}$ de retardos (k) óptimo en modelo VAR Table 3. Lag order selection in model VAR

\begin{tabular}{cccc}
\hline$k$ & EPF & Akaike & Schwarz \\
\hline 0 & $6,75 \mathrm{E}-05$ & $-1,090306$ & $-0,962340$ \\
\hline 1 & $1,12 \mathrm{E}-05^{*}$ & $-2,892102^{*}$ & $-2,380237^{*}$ \\
\hline 2 & $1,49 \mathrm{E}-05$ & $-2,615198$ & $-1,719434$ \\
\hline 3 & $2,00 \mathrm{E}-05$ & $-2,341849$ & $-1,062186$ \\
\hline 4 & $2,81 \mathrm{E}-05$ & $-2,047085$ & $-0,383523$ \\
\hline 5 & $4,01 \mathrm{E}-05$ & $-1,765574$ & 0,281886 \\
\hline 6 & $4,92 \mathrm{E}-05$ & $-1,677895$ & 0,753464 \\
\hline 7 & $7,07 \mathrm{E}-05$ & $-1,491134$ & 1,324124 \\
\hline 8 & 0,000103 & $-1,378466$ & 1,820691 \\
\hline
\end{tabular}

Fuente: Elaboración propia.

número de retardos $k$ a incluir en el modelo VAR debe ser 1, lo que implica que en el modelo de corrección de error (2) se incluyen 0 retardos.

En cuando a la componente determinista, se decide incluir en (2) una constante no restringida, además de una constante en las relaciones de cointegración, para recoger la tendencia determinista lineal que induce la presencia conjunta de raíz unitaria y término constante, detectada en las tres series al estudiar sus respectivos órdenes de integración.

\section{b) Estimación de las relaciones de cointegración}

Para determinar el número de relaciones de cointegración se aplicaron los contrastes de la traza y del autovalor máximo tomando en el modelo (2) $p=3, k=1$ y una constante no restringida. Los resultados de los contrastes bajo estas condiciones se recogen en la tabla 4 , de donde se concluye con ambos, para un nivel de significación del $5 \%$, que existen 2 relaciones de cointegración entre las series de precios. Por tanto, como se admite la existencia de $p-1=2$ vectores de cointegración, se cumple la condición necesaria para que los tres mercados estén perfectamente integrados. Asimismo, puesto que un número cada vez más elevado de vectores de cointegración implica una mayor estabilidad en el sistema, el resultado de dos de estos vectores entre las series de precios de aceite de oliva confiere a dicho mercado una gran fortaleza y estabilidad (según Dickey, Jansen y Thornton (1991), como los vectores de cointegración representan restricciones que un sistema económico impone en los movimientos a largo plazo de las variables de dicho sistema, un número cada vez mayor de tales vectores es visto como un aumento en la estabilidad del sistema).

La tabla 5 recoge las estimaciones de los parámetros de las dos relaciones de cointegración en las que se ha normalizado respecto del precio de Italia y Grecia, respectivamente, mientras que la tabla 6 presenta las estimaciones de los correspondientes coeficientes de ajuste. 
Tabla 4. Contraste de cointegración

Table 4. Testing for cointegration

\begin{tabular}{lccccc}
\hline N $\begin{array}{l}\text { Nelaciones } \\
\text { cointegración } \\
\left(H_{0}\right)\end{array}$ & \multicolumn{2}{c}{ Test de la traza } & \multicolumn{2}{c}{ Test del autovalor máximo } \\
\cline { 2 - 5 } & Autovalor & Estadístico & Valor crítico al 5\% & Estadístico & Valor crítico al 5\% \\
\hline Ninguna & 0,333870 & $34,92557 *$ & 29,68 & $33,59140 *$ & 20,97 \\
Al menos 1 & 0,279551 & $16,64337 *$ & 15,41 & $17,37130 *$ & 14,07 \\
Al menos 2 & 0,041103 & 1,888733 & 3,76 & 1,469176 & 3,76 \\
\hline
\end{tabular}

* Indica rechazo de la hipótesis nula al 5\% según valores críticos proporcionados por Mackinnon et al. (1999). Fuente: Elaboración propia.

Tabla 5. Estimación del espacio de cointegración (matriz $\beta$ )

Table 5. Estimating the cointegrating space (matrix $\beta$ )

\begin{tabular}{lcccc}
\hline & Matriz $\beta$ & \multicolumn{2}{c}{ Significación individual } \\
\hline Precio & Vector 1 & Vector 2 & Hipótesis & Estadístico \\
\hline España & $-0,511835$ & -0.48741 & $H_{0}: \beta_{11}=0, \beta_{12}=0$ & $28,27619 *$ \\
Italia & 1 & -0.472204 & $H_{0}: \beta_{21}=0, \beta_{22}=0$ & $18,00724 *$ \\
Grecia & $-0,483625$ & 1 & $H_{0}: \beta_{31}=0, \beta_{32}=0$ & $25,14901 *$ \\
Constante & $-0,181196$ & $-0,184492$ & & \\
\hline
\end{tabular}

Valor crítico (5\%): $\chi^{2}(2)=5,99$

(*) Indica rechazo de la hipótesis nula (6) al 5\%

Fuente: Elaboración propia.

La relevancia de cada serie de precios $\left(P_{i t}\right)$ en la relación a largo plazo se estudia mediante el contraste de nulidad sobre sus correspondientes coeficientes $\left(\beta_{i t}\right)$ en cada ecuación de cointegración. Los valores calculados del estadístico del contraste para cada una de las tres series incluidas en el modelo aparecen en la última columna de la tabla 5. Los resultados indican que las tres series de precios consideradas forman parte del espacio de cointegración.

Se analiza la exogeneidad débil de las tres series de precios $\left(P_{i t}\right)$, mediante el contraste de nulidad sobre sus correspondientes coeficientes $\left(\beta_{i t}\right)$ en cada ecuación de cointegración. Los valores calculados del estadístico del contraste para cada una de las tres series incluidas en el modelo, se presentan en la última columna de la tabla 6. Los resultados nos indican que tanto el precio de España como el de Italia son débilmente exógenos respecto al espacio de cointegración, lo que implicaría que los mercados correspondientes deberían ser considerados como líderes. Esto significa que estos dos precios participan en las relaciones de equilibrio detectadas pero no se ven influenciados por shocks en dichas relaciones.

Brester y Goodwin (1993) señalan que cuando existen $p$ - 1 vectores de cointegración entre 
Tabla 6. Estimación de los coeficientes de ajuste (matriz $\alpha$ ) y exogeneidad débil

Table 6. Estimating speed of adjusment parameters (matrix $\alpha$ ) and weak exogeneity

\begin{tabular}{lcccc}
\hline & \multicolumn{2}{c}{ Matriz $\alpha$} & \multicolumn{2}{c}{ Exogeneidad débil } \\
\hline Precio & Columna 1 & Columna 2 & Hipótesis & Estadístico \\
\hline España & 0,563176 & 0,5069371 & $H_{0}: \alpha_{11}=0, \alpha_{12}=0$ & 3,305756 \\
Italia & $-0,44638$ & $-0,118599$ & $H_{0}: \alpha_{21}=0, \alpha_{22}=0$ & 1,869319 \\
Grecia & $-0,337443$ & $-0,809371$ & $H_{0}: \alpha_{31}=0, \alpha_{32}=0$ & $8,075854^{*}$ \\
\hline
\end{tabular}

Valor crítico $(5 \%): \chi^{2}(2)=5,99$

$\left.{ }^{*}\right)$ Indica rechazo de la hipótesis nula al 5\%

Fuente: Elaboración propia.

$p$ series de precios, sólo uno puede considerarse como representativo de todo el sistema. Por ello, el resultado obtenido puede deberse a que el mercado internacional del aceite de oliva está segmentado en dos submercados, liderando España e Italia cada uno ellos, o bien que en realidad solo uno de ellos es el dominante. En cualquier caso, mediante un análisis más detallado del modelo de corrección de error especificado permitirá pronunciarse sobre qué opción es la más plausible (situación similar se presenta en el estudio de

$$
\begin{gathered}
\text { Alternativa } 1 \\
\beta^{\prime} Z_{t-1}=\left[\begin{array}{cccc}
* & 1 & 0 & * \\
* & 0 & 1 & *
\end{array}\right]\left[\begin{array}{c}
\text { LPcEspaña } \\
\text { LPcItalia } \\
\text { LPcGrecia } \\
\text { cte }
\end{array}\right]_{t-1}
\end{gathered}
$$

Las estimaciones de las matrices $\alpha$ y $\beta$ de cada alternativa se muestran en las tablas 7 y 8 , respectivamente. En ambos casos, existen claros indicios de cumplimiento de la paridad $(1,-1)$ entre las diferentes parejas de precios, ya que los coeficientes estimados de ambos vectores de cointegración para España en la alternativa 1 e Italia en la 2 , se muestran transmisión de precios en los mercados españoles de ovino de Boshnjaku et al. (2003))

\section{c) Identificación de las relaciones de cointegración}

Teniendo en cuenta los resultados obtenidos, se plantean dos identificaciones alternativas. Para la primera, los vectores de cointegración se normalizan respecto de Italia y Grecia, mientras que para la segunda la normalización se realiza respecto de España y Grecia

\section{Alternativa 2}

$$
\beta^{\prime} \mathrm{Z}_{t-1}=\left[\begin{array}{cccc}
1 & * & 0 & * \\
0 & * & 1 & *
\end{array}\right]\left[\begin{array}{c}
\text { LPcEspaña } \\
\text { LPcItalia } \\
\text { LPcGrecia } \\
\text { cte }
\end{array}\right]_{t-1}
$$

muy próximos a -1. Para confirmar este extremo, es decir, para admitir la perfecta integración a largo plazo de los tres mercados se contrasta si en las dos ecuaciones de cointegración se pueden aceptar las relaciones de la forma $(1,-1)$ entre las diferentes parejas de series de precios. En este caso, la hipótesis nula a contrastar podría ser 


$$
\beta^{\prime}=\left[\begin{array}{llll}
-1 & 1 & 0 & * \\
-1 & 0 & 1 & *
\end{array}\right] \quad \text { ó } \quad \beta^{\prime}=\left[\begin{array}{llll}
1 & -1 & 0 & * \\
0 & -1 & 1 & *
\end{array}\right]
$$

siendo el valor calculado del estadístico para este contraste 2,16 con un $p$-valor de 0,338842. Por tanto, no se rechaza la hipótesis nula, lo que permite afirmar que se cumple la condición suficiente para afirmar que los mercados considerados están perfectamente integrados, es decir, a largo plazo, los precios en los tres mercados se mueven en la misma dirección existiendo una única tendencia común que condiciona la evolución de los precios.

Ahora bien, en la alternativa 1, el precio de Italia resulta ser débilmente exógeno res- pecto del espacio de cointegración (sus coeficientes en la matriz $\alpha$ no son significativos). En realidad, tan solo el precio de Grecia reacciona ante desviaciones en el nivel de equilibrio del segundo vector de cointegración (relación entre España y Grecia). En cambio, cuando se toma como referencia el precio de Italia (alternativa 2) se observa cómo los precios de España y Grecia reaccionan ante desviaciones en el nivel de equilibrio, mientras que el precio de Italia se muestra débilmente exógeno. En principio, teniendo en cuenta que Italia es el primer país exportador de aceite envasado, lo más razonable es pensar que Italia es el mercado dominante.

Tabla 7. Estimación del espacio de cointegración (Alternativa 1: España líder) Table 7. Estimating of the cointegrating space (Alternative 1: Spain leader)

\begin{tabular}{lcccc}
\hline & \multicolumn{2}{c}{ Matriz $\beta$} & \multicolumn{2}{c}{ Matriz $\alpha$} \\
\hline Precio & Vector 1 & Vector 2 & Columna 1 & Columna 1 \\
\hline España & $-0,968804$ & $-0,944883$ & 0,323798 & 0,234571 \\
& & & $(0,96506)$ & $(1,08139)$ \\
Italia & 1 & 0 & $-0,39037$ & 0,097281 \\
& & & $(-1,3834)$ & $(0,53324)$ \\
Grecia & 0 & 1 & 0,044746 & $-0,646176$ \\
& & & $(0,12426)$ & $(-2,77556)^{*}$ \\
Constante & $-0,350454$ & $-0,349978$ & & \\
\hline
\end{tabular}

Entre paréntesis se muestra el $t$-ratio asociado a cada coeficiente estimado de la matriz $\alpha$

${ }^{*}$ ) Indica significación del coeficiente al 10\%.

Fuente: Elaboración propia.

No obstante, los resultados anteriores solo eran orientativos, por lo que se debía comprobar si se mantiene el liderazgo de Italia en un contexto de prefecta integración de los mercados. Así, el contraste simultáneo de las hipótesis integración perfecta y exogeneidad débil del precio de Italia lleva aceptar ambas hipótesis de manera simultánea ( $p$-valor $=0,421583$ ). 
Tabla 8. Estimación del espacio de cointegración (Alternativa 2: Italia líder)

Table 8. Estimating of the cointegrating space (Alternative 2: Italy leader)

\begin{tabular}{lccrr}
\hline & & Matriz $\beta$ & & \multicolumn{2}{c}{ Matriz $\alpha$} \\
\hline Precio & Vector 1 & Vector 2 & Columna 1 & Columna 1 \\
\hline España & 1 & 0 & $-0,535339$ & 0,234571 \\
& & & $(-1,70411)^{*}$ & $(1,08139)$ \\
Italia & $-1,0322$ & $-0,975309$ & 0,28628 & 0,097281 \\
& & & $(-1,083539)$ & $(0,53324)$ \\
Grecia & 0 & 1 & 0,567211 & $-0,646176$ \\
& & & $(1,6823)^{*}$ & $(-2,77556)^{*}$ \\
Constante & 0,361739 & $-0,008177$ & & \\
\hline
\end{tabular}

Entre paréntesis se muestra el $t$-ratio asociado a cada coeficiente estimado de la matriz $\alpha$

${ }^{*}$ ) Indica significación del coeficiente al $10 \%$.

Fuente: Elaboración propia.

Relaciones dinámicas a corto plazo

Una vez analizado el largo plazo para el mercado considerado, se estudian las relaciones dinámicas a corto plazo. Como las series de precios implicadas en dicho mercado están cointegradas es preferible realizar el análisis mediante las Funciones Impulso-Respuesta Generalizadas (FIRG). Así, las figuras 3 (a)-(c) recogen las respuestas de las series de precios ante un shock no previsto en cada una de ellas imponiendo el cumplimiento de la transmisión perfecta entre los precios y que Italia es un mercado líder.

En primer lugar, ante un shock inesperado en el precio de España (Figura 3b) las respuestas del propio precio y del precio de Grecia son positivas e inmediatas, siendo de mayor magnitud la del precio español en el primer año. A partir del segundo año la respuesta griega es ligeramente superior a la española para acabar ambas alcanzando el equilibrio a partir del $5^{\circ}$ año.

Por otro lado, un shock en el precio de Grecia (Figura 3c) genera una reacción positiva e inmediata de gran magnitud en el propio precio y de menor magnitud en el de España. Como en el caso anterior, transcurridos 5 años las respuestas se estabilizan en la tendencia que marca el precio de Italia.

Finalmente, hay que reseñar que la respuesta de Italia ante shocks inesperados en los precios de cada mercado es claramente de mayor magnitud en el caso de un shock en el propio precio italiano. En los otros dos casos, la respuesta es de menor magnitud, pudiendo llegar a ser estadísticamente no significativa, lo que refuerza la consideración de Italia como mercado líder. Más concretamente, un posible shock en el precio de Italia, como muestra la Figura 3a, genera una reacción positiva e inmediata sobre los precios de España y Grecia, con tendencia a estabilizarse a partir del $5^{\circ}$ año. Esto significa que una variación inesperada en el precio de Italia tiene un efecto permanente en el resto de mercados. Por tanto, es el precio de Italia el que marca la tendencia común que, de acuerdo con los resultados del análisis de cointegración, existe entre los precios de los tres mercados, lo que refuerza su posición de liderazgo. 


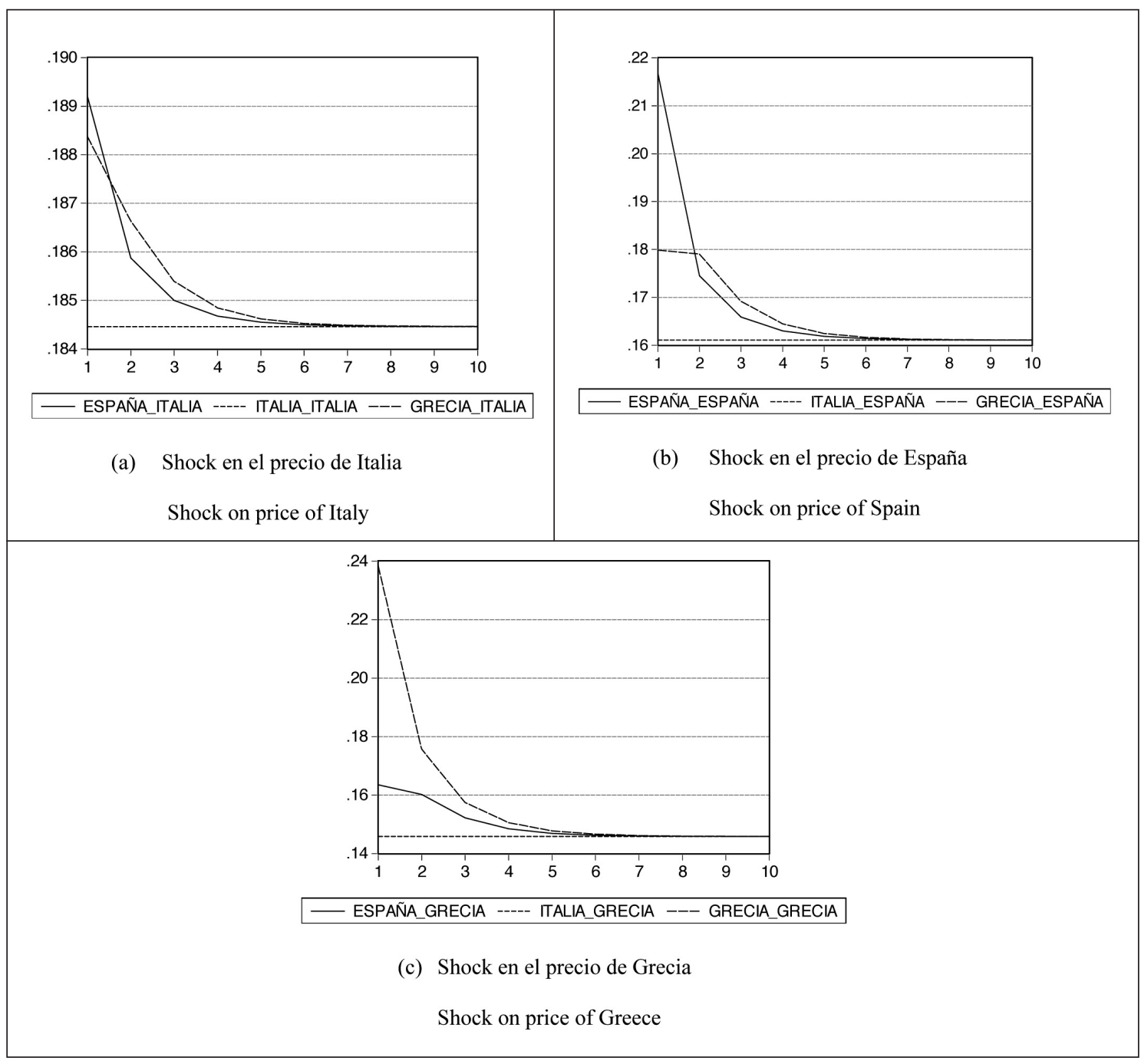

Figura 3. Shock en el precio en cada uno de los países.

Figure 3. Shock on the prices.

\section{Conclusiones}

El estudio de las series de precios de aceite de oliva realizado en este trabajo de los tres principales mercados de producción y exportación mundiales (España, Italia y Grecia), ha permitido comprobar su deambular alrededor de una tendencia lineal creciente a lo largo del período considerado (1961 a 2007). El análisis de las mismas ha permitido concluir que dicha tendencia se debe a la presencia de una raíz unitaria y un término constante en el mecanismo generador de cada serie, lo que implica ausencia de tendencias deterministas explícitas en dicho mecanismo. 
En el aspecto del comportamiento conjunto se han encontrado dos relaciones de cointegración entre las tres series de precios. Esto representa de un alto grado de integración que confiere al mercado una gran fortaleza y estabilidad y es indicativo de que los mercados son globalmente eficientes, no solo de forma interna sino también en cuanto a sus posibles interrelaciones. Esta eficiencia global se traducirá en que, a largo plazo, los precios en los diferentes mercados se mueven en la misma dirección existiendo una única tendencia común.

Asimismo, se comprueba que la integración entre los mercados es perfecta, pues las diferentes parejas de precios verifican la paridad $(1,-1)$. Esto supone una transmisión completa, a largo plazo, de los precios entre los tres mercados, es decir, ante los cambios en alguno de los tres precios, los demás reaccionarán igualándose (deducidos los costes de transferencia) a largo plazo, de forma que las desviaciones con respecto a la situación de equilibrio se pueden considerar transitorias. En este contexto de integración perfecta, el precio de Italia resulta exógeno débil, lo que lo sitúa como mercado dominante. Esto indica que el precio de Italia no se ve afectado por shocks inesperados en los precios de España y Grecia.

Así, de un lado podemos señalar la presencia de un arbitraje perfecto a largo plazo, el cual actúa de cierta manera como un seguro de estabilidad en los precios, amortiguando las importantes oscilaciones que serían de esperar en un producto como el aceite, donde fenómenos como la vecería o los años de sequía dan lugar a una producción variable en cada uno de los principales países productores.

De otro, la constatación del liderazgo de Italia en la formación de precios del aceite en el mercado internacional lleva a plantearse la necesidad de una revisión de las estrategias comerciales del sector español, que no ve que su enorme liderazgo en la producción mundial de lugar a un peso equivalente en la formación del mercado mundial, ya que se da la paradoja que nuestro principal cliente es a su vez nuestro principal competidor en el mercado mundial, y es gracias a esta compras de aceite español con las que Italia obtiene su preponderancia. Esta situación ha venido dada en parte por el importante aumento de producción que se ha dado en España en las últimas dos décadas, donde el principal objetivo de las productoras de aceite ha sido en muchas ocasiones más encontrar un cliente al que asegurar colocar el producto que ejercer de líder en los mercados, habiéndose creado un estructura de mercado doblemente oligopsonista: la gran distribución nacional (Langreo, 2010) y las envasadoras italianas.

Son las políticas comerciales de diferenciación de productos y de calidad la gran baza de competencia directa del aceite español (Dios y Martínez-Paz, 2011). La promoción internacional de las Denominaciones de Origen españolas como sinónimo de calidad (Mesías et al., 2012) frente a las ventas de aceites mezclados que realiza el país transalpino. A su vez una regulación más rigurosa de las políticas comunes de etiquetado en la UE para este producto sería también un elemento útil para esta línea de acción de promoción del aceite de oliva español.

De otro lado la colaboración específica de la pequeña y media industria extractiva de aceite de oliva puede conseguir la generación de ventajas competitivas dinámicas (Moral y Lanzas, 2010) para rentabilizar el importante potencial del mercado internacional, que también debería contar con el apoyo específico de las administraciones públicas para su entrada en los mercados emergentes, como ya hizo el Plan de Promoción Internacional del Aceite de Oliva Español en 2011, con importantes y exitosas campañas en Estados Unidos, China, Australia o los países de la Europa del Este, entre otros. 


\section{Bibliografía}

AAO, 2013. Agencia para el Aceite de Oliva. Disponible en http://aplicaciones.magrama.es/ pwAgenciaAO/ (7 abril 2013).

Abdulai A, 2000. Spatial price transmission and asymmetry in the Ghanaian maize market. Journal of Development Studies 63: 327-349.

Alimarket, 2011. El aceite de oliva español bate un nuevo récord de exportación. Alimarket. Noticia. Aceites. 27 octubre 2011.

Alonso J, Arcila A, 2012. Un modelo de predicciones diarias para contratos de futuros de azúcar. Revista Economía \& Region 6 (2): 33-51.

Antelo, $A B, 2012$. Aceites: el precio condiciona el desarrollo del sector. Alimarket 257: 76-93.

Baffes J, Ajwad M, 2001. Identifying price linkages: a review of the literature and an application to the world market of cotton. Applied Economics 33: 1927-1941.

Barrett C, 2001. Measuring integration and efficiency in international agricultural markets. Review of Agricultural Economics 23: 19-32.

Barrett C, Li J, 2002. Distinguishing between equilibrium and integration in spatial price analysis. American Journal of Agricultural Economics 84: 292-307.

Ben Kaabia M, Gil, JM, 2008. Modelling import demand systems with nonstationary data: an application to the French imports of virgin olive oil. European Association of Agricultural Economists, 107th Seminar, Sevilla, Spain.

Boshnjaku L, Ben-Kaabia M, Gil JM, 2003. Transmisión de precios en los mercados regionales de ovino en España. Economía Agraria y Recursos Naturales 3(1): 71-103.

Brester G, Goodwin B, 1993. Vertical and Horizontal Price Linkages and Market Concentration in the U.S. Wheat Milling Industry. Review of Agricultural Economics 15(3): 507-519.

Dickey D, Jansen D, Thornton D, 1991. A primer on cointegration with an application to money and income. Federal Reserve Bank of St. Louis Review 73: 58-78.
Dios R, Roldán J, 2006. A strategy for testing the unit root in $A R(1)$ model with intercept. $A$ Monte Carlo experiment. Journal of Statistical Planning and Inference 136(8): 2685-2705.

Dios-Palomares R, Martínez-Paz JM, 2011. Technical, quality and environmental efficiency of the olive oil industry. Food Policy 36(4): 526-534.

Engle R, Granger C, 1987. Co-integration and error correction: representation, estimation and testing. Econometrica 55: 251-276.

Fackler, P, Goodwin, B, 2001. Spatial price analysis. In Gardner B, Rausser B, Handbook of Agricultural Economics, vol. 1B, ed. Amsterdam: Elsevier.

FAO, 2012. Food and Agriculture Organization statistical database. Disponible en http://faostat.fao.org/ (20 julio 2012).

Gil JM, Clemente J, Montañes A, Reyes M, 1996. Integración espacial y cointegración: una aplicación al mercado de cereales en España. Estudios de economía aplicada 6: 103-130.

Gil JM, Dhehibi B, Kaabia M, Angulo A, 2004. Non-stationarity and the import demand for virgin olive oil in the European Union. Applied Economics 36: 1859-1869.

Goodwin B, Schroeder T, 1991. Cointegration tests and spatial price linkages in regional cattle markets. American Journal of Agricultural Economics 73(2): 452-464.

Gutiérrez M, Vega M, 2009. La dinámica del mercado de origen de los aceites de oliva: una aproximación a partir de la teoría de la cointegración. Consejo Económico y Social de la Provincia de Jaén, Jaén, 120 pp.

Gutiérrez M, 2012. La transmisión de precios en la cadena agroalimentaria: el mercado español de los aceites de oliva. Tesis doctoral. Universidad de Jaén. 440 pp.

ICEX, 2012. Información sectorial de alimentos: aceite de oliva. División de Productos Agroalimentarios, Instituto Español de Comercio Exterior, Madrid. http://www.icex.es

IOC, 2012. International Olive Council. World Olive Oil Figures http://www.internationaloliveoil.org/ 
Johansen S, 1988. Statistical analysis of cointegration vectors. Journal of Economic Dynamics and Control 12: 231-254.

Johansen S, 1995. Likelihood-based Inference in Cointegrated Vector Autoregressive Models. Oxford University Press.

Koehler A, Murphree E, 1988. A Comparison of the Akaike and Schwarz Criteria for Selecting Model Order. Journal of the Royal Statistical Society, Series C, 37: 187-195.

Langreo, A, 2002. Los mercados de graneles de aceite de oliva en España. Distribucion y Consumo, Noviembre-Diciembre: 108-117.

Langreo A, 2010. La estrategia empresarial en el sector del aceite de oliva y su evolución desde la transición política. Revista de Estudios Empresariales. Segunda época. 1: 7-31.

Lanzas J, Moral E, 2010. Mercados estratégicos para la promoción del aceite de oliva virgen español. Revista de Estudios Empresariales. Segunda época. 1: 85-102.

Mackinnon JG, Haug A, Michelis L, 1999. Numerical distribution functions of likelihood ratio tests for cointegration. Journal of Applied Econometrics 14(5): 563-577.

MAGRAMA, 2013. Avances de superficies y producciones de cultivos: Enero 2013. Ministerio de Agricultura, Alimentación y Medio Ambiente. Madrid.

Martínez-Carrasco F, 2004. Selection of marketing channels by intensive horticultural crop growers in Almería. Spanish Journal of Agricultural Research 2 (1): 27-33.

Mesias-Díaz, F, Martínez-Carrasco F, Martínez-Paz JM, Gaspar P, 2012. Consumer knowledge, consumption, and willingness to pay for organic tomatoes. British Food Journal 114 (3): 318-334.
Nielsen, M, 2005. Price formation and market integration on the European first-hand market for whitefish. Marine Resource Economics 20: 185-202.

Parras M, Senise O, Torres F, 2003. Comercialización de aceites de oliva y concentración de la oferta: estado de la cuestión. Boletín del Instituto de Estudios Giennenses 185: 355-372.

Parras-Rosa M, Vega-Zamora M, Torres-Ruiz F, Murgado-Armenteros E, Gutiérrez-Salcedo $M$, 2013. Posicionamiento de envases en el mercado del aceite de oliva virgen extra: un estudio exploratorio. Información Técnica Económica Agraria 109 (1): 107-123.

Rapsomanikis G, Hallam D, Conforti1 P, 2004. Market integration and price transmission in selected food and cash crop markets of developing countries: review and applications in FAO, Commodity Market Review 2003-2004 51-77. FAO. Roma.

Roldan J, Dios R, 2008. Ley de precio único en el mercado español del aceite de oliva. Economía Agraria y Recursos Naturales 8(1) 37-55.

Roldán J, 2000. Análisis sobre la detección de raíces unitarias desde la perspectiva de la no similaridad. Estudio de integración en el mercado del aceite de oliva. Tesis doctoral, Universidad de Córdoba.

Sanjuán A, Gil J, 2001. Price transmission analysis: a flexible methodological approach applied to European pork and lamb markets. Applied Economics 33: 123-131.

Silvapulle P, Jayasuriya S, 1994. Testing for Philippines rice market integration: a multiple cointegration approach. Journal of Agricultural Economics 45(3): 369-380.

(Aceptado para publicación el 26 de abril de 2013) 